\title{
La psicopatología insustancial en la era del DSM IV y la CIE-10
}

RESUMEN: Se comentan las propuestas de un artículo de reciente aparición en esta revista, que critica algunas concepciones de la psicopatología encaminadas a postular a ésta como base científica para las prácticas profesionales de psiquiatras y psicólogos.

Se expone el concepto de práctica profesional en relación con el contexto social y tecnológico en el que ésta se desarrolla, ejemplificándolo en la situación actual con respecto a los manuales psicopatológicos DSM-IV y CIE-10. PALABRAS CLAVE: Psicopatología, Práctica profesional, Actitud ética, Evolución técnica.

\begin{abstract}
We write about the proposals of an article published in this journal recently. This paper critics the idea of a psychopatology designed as scientific basis for professional practices of psychiatrists and psychologists.

We develop the concept of professional practice relationed with the social and technological context where it is. We exemplify it describing the present situation about the DSM-IV and ICD-10 psychopatological manuals.

KEY WORDS: Psichopatology, Professional practice, Ethical attitude, Technical evolution.
\end{abstract}

En el número de la revista de la AEN correspondiente al período OctubreDiciembre de 2001, en la sección Debates, se publica un artículo del Dr Fernández Liria titulado De las psicopatologías críticas a la crítica de la psicopatología (1). En el mismo, y a propósito de una posible crítica general a la psicopatología, el mencionado Dr. abunda en una línea de pensamiento que, de diversas formas, viene sosteniendo desde hace tiempo (2). Si no entiendo mal, lo esencial de su argumentación se apoyaría en la hipótesis de que en el síntoma psiquiátrico (o psicológico) no hay nada de sustancial y que la crítica a la psicopatología que en esta concepción subyace nos debería llevar a un cambio de objeto de interés epistemológico. Lo que ahora habría que mostrar es, no aquella sustancia (química o psicológica, por ejemplo) que está detrás del síntoma (vale decir síndrome o enfermedad) y que sería causa del mismo, sino "el proceso por el que se produce la aparente sustanciación del síntoma al servicio de una organización social de las relaciones de ayuda" (1). Subrayo lo de aparente, porque bajo la connotación de espejismo o engaño que el adjetivo trasluce, parece asomar la ominosa sospecha, de que tras aquél podría no haber nada, o por lo menos nada sustantivo.

Rev. Asoc. Esp. Neuropsiq., 2002, vol XXII, n. ${ }^{\circ} 82$, pp. 67-73 
Frente a cualquier posible psicopatología crítica $(3,4,5,6,7)$, encaminada a sostener la vigencia de éésta como ciencia (sea manteniendo propuestas descriptivas, cognitivas o hermenéuticas $(8,9)$, la crítica de la psicopatología (de cualquier psicopatología) sostendría que "no hay en la naturaleza síntomas o enfermedades esperando a ser descubiertas por psicopatólogos" (1). La sustancia de la enfermedad mental se nos escapa, quizáá porque no existe o, tal vez, porque la buscamos en un lugar inapropiado. Lo que se nos propone es que "los síntomas y las enfermedades son constructos" (1) y que "la distinción entre distintas enfermedades adquiere sentido en la medida que sirve para poner en marcha distintos procedimientos y para hacer predicciones sobre cuáles serán los resultados obtenidos con éstos" (1). Es decir, lo que existiría, más que diversas entidades nosológicas muy bien definidas, es una serie de prácticas (diagnósticas, terapéuticas, de construcción teórica o reclamadas por la sociedad) de las que hay que dar cuenta y en torno a las que hay que organizar no sólo el despliegue de nuestros servicios, sino también la articulación de nuestro pensamiento psiquiátrico, si queremos mantener la posibilidad de hacer de él, alguna vez, algo asimilable a una ciencia.

Si lo anterior es cierto, entonces "el síntoma, la enfermedad es también función del proceso de intervención. Las psicopatologías tal y como las conocemos, ocultan este hecho importante como ocultan que es un contexto cultural el que le otorga a los fenómenos el carácter de morbosos" (1). La crítica a la psicopatología heredada es, pues, radical ya que, tras más de cien años de historia (3), y lejos de haber conseguido delimitar un territorio epistemológico propio, con lo que nos encontramos (como algunas veces se ha dicho) es con un continuo vaivén de ensanchamiento o reducción, discusión y redefinición de este espacio mismo, donde entran y salen entidades, conceptos y clasificaciones en función, no de nada esencial, sino de nuevos instrumentos de intervencióón o nuevos consensos sociales. Lo radical de la crítica que propone el Dr. Fernández Liria, consiste en no escandalizarse demasiado por este hecho o por la muestra de debilidad que supone respecto al núcleo duro de una posible ciencia psicopatológica. "Probablemente es más sensato que consideremos que esto es... la naturaleza misma de la disciplina" (1).

Así, pues, nada más que humo y paja. Sólo hay prácticas sociales, no hay psicopatología sustantiva. Esta, como mucho, podría aspirar a ser "adjetiva", esto es, a vivir sin nombre propio o de una forma insustancial.

Tengo que reconocer que, desde hace años, esta crítica me atrae, como sólo pueden atraer las aventuras peligrosas : de una manera fatal. Veo en ella el punto de partida para construir, desde una posición coherente, una teoría (o teorías) sobre "el objeto psíquico" (cualquier cosa que esto sea) lo más científica posible, a partir de nuestras prácticas profesionales (sean éstas teóricas, clínicas, organizativas, etc...), de su contexto, de sus avatares, de sus cambios (10). Esto aún no se ha intentado seriamente en ninguna parte y se me impone como esencial a fin encontrar una articulación entre aquello que (realmente) hacemos y sus fundamentos epistemológicos. Por otro lado, parece una exageración tirar por la borda, sin más, el cúmulo de observaciones, preguntas, hipótesis, teorías, tratados y propuestas con los que la psicopatología clásica nos acerca a un -hoy más 
imprescindible que nunca- ejercicio crítico del saber y las habilidades profesionales en psiquiatría $(8,9)$.

Espero que mi amigo el Dr. Fernández Liria, no proponga todavía la abolición de la psicopatología (clásica o moderna) y que -como a mí me gustaría- le demos a ésta aún tiempo para intentar resolver sus problemas metodológicos y encontrar alguna sustancia a la que agarrarse. A fin de cuentas, su posible objeto epistemológico, tampoco se ha demostrado como una completa y vana ilusión. Y todavía demasiadas cosas parecen depender del mantenimiento de esta estrategia de conocimiento. Sin embargo, a él y a mí, parecen preocuparnos otras direcciones del pensamiento y a ello nos aplicamos. Entonces sigamos con la crítica, en la espera de que no se molesten, ni se revuelvan demasiado nuestros queridos psicopatólogos.

La verdad es que las sucesivas ediciones del DSM, sobre todo a partir de la tercera, así como las correspondientes de la CIE, han venido a traer más concordia que discordia entre las huestes psiquiátricas y, también, entre éstas y "los otros", sean éstos usuarios, resto de profesionales de la sanidad, administradores, laboratorios o editores de revistas de prestigio.

Para empezar yo diría que, más que clasificaciones ateóricas (que lo son), lo son por multiteóricas hasta la exasperación. Se trata de los únicos consensos posibles que se pueden hacer en el estado actual (algo caótico) de nuestro conocimiento. Y si no de los únicos (puede haber revisiones a fondo de los actuales manuales o maneras distintas de clasificar) sí de la única forma posible de hacerlo hoy : esto es, mediante el consenso. Dicho sea de paso, el consenso es la manera menos científica, entre las catalogadas como científicas, de desarrollar una disciplina. Pero se trata, primero, de huir de la algarabía y, luego, si se puede, de alcanzar la ciencia. Además, y a pesar de todo, los actuales DSM y CIE no dejan de ser el lugar donde ha venido a depositarse lo más práctico y aprovechable (lo más disponible) de nuestro conocimiento psicopatológico. Tierra de aluvión, tributaria de nutrientes de río arriba, sometida a pérdidas, erosiones, meandros y corrientes, pero -después de todo- tierra de depósito.

Se trata -ya lo sabemos- de un mínimo común, que sólo satisface a unos pocos formalistas sin sustancia, que puede generar una pseudopsicopatología de "recetario diagnóstico" entre las nuevas generaciones de profesionales y que corre el riesgo de anular la observación y obturar la crítica del conocimiento y su desarrollo creativo. Pero se trata también, nada menos, que de un mínimo común. Porque, respóndanme con sinceridad ¿Qué había antes? Adelantaré mi respuesta : psicopatologías (en plural), escuelas, psiquiatrías nacionales, etc... Buenas allí donde había buenos maestros o tradiciones, malas o peores allí donde sólo había imitaciones o imposturas y nulas en la mayoría de los lugares, países, hospitales... (¿habrá que recordar los manicomios?, ¿habrá que recordar las prácticas entonces usuales y sus fundamentos, tan contemporáneos de la psicopatología clásica?). Ya sé que algo de eso puede pasar, y de hecho pasa también ahora, pero hoy en día -y porque existe ese mínimo común y porque es público y "obligatorio"- se puede realizar la denuncia y establecer una estrategia de cambio, quizá mínima, pero para todos, en todos los lugares, en todos los países. 
¿Por qué un consenso? ¿Por qué precisamente ahora? Porque a falta de demostraciones fehacientes (de carácter etiopatogénico, por ejemplo) había que frenar el disenso, por cuestiones prácticas e intereses compartidos, y no existía otro método mejor. Porque hace tiempo que estamos en la era de la globalización y hay que atender, de la mejor manera posible, al mayor número de personas. Además hay que hacerlo también fuera de los hospitales psiquiátricos y por motivos de demanda nuevos añadidos a los viejos. Todo esto exigía un acuerdo básico en cuanto a las formas de proceder, empezando por el diagnóstico (que se supone debe de inspirar a aquéllas). Desde el final de la Segunda Guerra Mundial, no sólo han aparecido tecnologías nuevas (psicofármacos, psicoterapias), no sólo se ha desarrollado la industria multinacional de la farmacia, que ha ejercido sus presiones sobre los profesionales de la psiquiatría y generado grandes conflictos de interés. También se han producido: 1) la universalización de la asistencia sanitaria (incluyendo la psiquiátrica en los países desarrollados) a cargo de los poderes públicos y como elemento de cohesión social; 2) un cierto avance en los derechos de las personas, empezando por el de disponer de su propia vida (incluso frente al criterio de su médico); 3) la aceptación generalizada de la necesidad de una mínima evidencia científica como sustento primordial de las prácticas profesionales en el campo médico; 4) la racionalización de los procesos diagnósticos y terapéuticos encaminada a una disminución de la variabilidad clínica y el consiguiente ahorro de costes y de disgustos.

$\mathrm{Ni}$ en medicina, ni en psiquiatría, nada de esto se puede hacer sin un corpus teórico central (pactado, acordado) con el que construir y dar un sentido básico a las prácticas profesionales se hagan éstas donde se hagan. Se trata de encontrar el fundamento a una clínica lo más común posible, si se quiere algo funcionarial y menos creativa, pero realizada en condiciones de realidad cotidiana por la mayoría de los profesionales, con la mayoría de los pacientes y en la mayoría de los lugares. Y no por un (real o supuesto) maestro, en una clínica modelo y con un paciente "de los de libro". A esto, y no a otra cosa, se le llama efectividad.

Pero nuestros manuales "de mínimos" tienen más utilidades. Han servido para mejorar la comunicación "interna" y "externa". Una cierta unificación terminológica era necesaria para nuestra entrada, con todos los derechos, en el universo médico, pero también para aclararnos entre nosotros y poder transmitir los conocimientos básicos a nuestros epígonos. Los administradores, quienes se supone que responden del dinero público, nuestros pacientes y sus familias estaban en su derecho de poder entender lo que decimos y ejercer sus controles. Gracias a su intento de precisión semántica los manuales (diagnósticos y estadísticos, recuérdese) también han servido de base a una estandarización que ha permitido realizar mediciones, estimación de necesidades y algunas predicciones. Estoy hablando, naturalmente, de epidemiología, que -como se sabe- es una importante rama de la salud pública moderna y que, hasta hace bien poco, era un terreno imposible para nuestra disciplina.

Puede ser cierto que la profusión de categorías clínicas actuales, y algunos de los constructos diagnósticos que manejamos, resulten una traba para una investigación que posibilite un auténtico avance en nuestro conocimiento clínico. Pero al menos están 
sirviendo para señalar mejor algunas zonas especialmente problemáticas (tanto antes como ahora) y activar a diversas escuelas psicopatológicas $(8,9)$, aunque sólo sea por mero reflejo defensivo. El desarrollo de la psiquiatría en la atención primaria (otro campo nuevo para la praxis y uno en los que más se resienten las nosografías presentes y pasadas) ha reavivado los debates sobre el concepto de caso y su frontera con la (supuesta) normalidad, así como sobre los diversos modelos teóricos disponibles para explicar la clínica de la somatización, por ejemplo, o la delimitación de los síntomas de las neurosis y su diferenciación de otras entidades. Por no hablar de las dudas sobre la propia realidad de las neurosis como entidades distintas.

Quizá ahora los maestros y genios de la profesión corran el riesgo de sentirse incomprendidos y solos. Pero con lo que hemos acabado es con la arbitrariedad camuflada de saber. Y es que se ha pasado, también aquí, de un modelo paternalista y beneficiente (de la teoría, de la clínica, de la enseñanza) a un modelo autonomista, donde el usuario (ya no sólo paciente) tiene derechos. El primero, el de conocer y entender su diagnóstico y posible tratamiento. Consecuentemente los principios éticos de "No Maleficiencia" y "Justicia" han pasado a primar. Se trata, por el primero, de garantizar las prácticas mínimas exigibles, en cualquier lugar, como adecuadas a buen fin terapéutico. Se trata, por el segundo, de su aplicación no discriminada, puesto que todo el mundo que necesite asistencia está en su derecho de pedirla y de se que se le haga efectiva.

Resulta imposible no encontrar la relación práctica entre esa "psicopatología de mínimos" y ese "primun non nocere" o primacía del no hacer daño sobre el hacer "el bien" a todo trance. Se acabó el furor terapéutico y la aplicación de "lo de siempre", "lo que aprendí con mi maestro", "lo último" o "lo que más me convence a mí", sin atenerse a más criterio. Estandarizar, medir y someter los procedimientos a verificación : una auténtica plaga para los creadores indiscriminados. Pero es que, ahora, hay algunas (aunque incipientes) terapéuticas y casi todas tienen sus efectos colaterales.

Estoy convencido de que, en el terreno psicopatológico, los DSM y la CIE son una estación intermedia que hay que superar. Sus limitaciones son claras y sólo pueden aceptarse de una manera provisional y a falta de algo mejor, que satisfaga todas y cada una de las condiciones necesarias. Pero, eso sí, que las satisfaga, porque lo anterior tampoco lo hacía. Algunas de esas condiciones, que son las que delimitan la única praxis posible hoy en día para las profesiones de lo "psi”, ya han sido mencionadas arriba. De aquí sólo podemos salir por la vía legítima, que es la de la investigación seria y el compromiso con nuestros pacientes y nuestros conciudadanos (los que realmente hay, no los que nos gustaría que hubiese) y que son, también -como nosotros- consumidores. Es lícito aún tener opciones de escuela, pero ésas se han de guardar para investigar, estudiar y trasmitir el conocimiento (en sana competencia con otros) y no pueden excusar de incumplir los mínimos establecidos por el suelo común de lo poco que sabemos.

La pregunta final es un poco molesta, pero es una consecuencia lógica de lo anterior y que otras profesiones médicas ya se han planteado: ¿Hace falta saber mucha psicopatología para ser un buen psiquiatra? La respuesta a esta pregunta es la misma que los médicos de hoy han de darse si se preguntan por cuántos detalles clínicos, de anatomía 
patológica o de fisiología necesitan saber para atender bien a sus pacientes. La respuesta no es necesariamente positiva y depende del para qué de la práctica a considerar. Cierto es que el saber -sobre todo si ayuda a la crítica- nunca viene mal, pero no puede exigirse la excelencia en condiciones estándar y de eso hablamos.

Así pues, y mientras esperamos a ver si la psicopatología responde o no a algunas de nuestras preguntas fundamentales, el análisis de las prácticas de nuestra profesión y el estudio del contexto (social, cultural) que las condiciona pueden ayudarnos a aclarar algunas zonas oscuras que se imponen ante nosotros (10). La historia de las diversas formas institucionales de tratar los problemas psiquiátricos, la historia de los términos con los que los denominamos y entendemos (locura, enfermedad, trastorno, malestar...), es la historia de nuestras actividades con nuestros pacientes y con nuestros colegas y la historia de los lugares en los que socialmente se acepta (o se nos pide) que las realicemos o que las transmitamos como saber. No es imposible que unas prácticas como la nuestras creen el objeto sobre el que teorizan (no sabemos aún si en todo o sólo en parte). Pero, independientemente de ello, el estudiarlas ayudará a elucidar la naturaleza de aquello que, desde los clásicos griegos, llamamos psique.

Ya Foucault (11) sospechó que la psicopatología podría ser una "pseudociencia". No dijo que su intento fuera vano o que no pudiera dar lugar a una ciencia, o que no sirviera para nada. Lo que dijo es que, como la economía, como la historia natural, la psicopatología podría funcionar de otra manera. Podría constituir un cuerpo relativamente autónomo de saber, organizado en torno a unos enunciados, que se articulan entre sí, y que conforman unos modos y unos emplazamientos según los cuales se establece cómo integrar a lo ya dicho todo enunciado nuevo. Pero, para este autor, saber no equivale necesariamente a ciencia. De lo que se trata es de estudiar la adecuada relación entre ambas categorías y de construir criterios para verificar cuándo una determinada disciplina atraviesa los sucesivos umbrales de positividad, de epistemologización, de cientificidad y de formalización, propios de las ciencias desarrolladas (11).

"Ocuparse del funcionamiento ideológico de una ciencia para hacerlo aparecer o para modificarlo, no es sacar a la luz los presupuestos filosóficos que pueden habitarla; no es volver a los fundamentos que la han hecho posible y que la legitiman: es volver a ponerla en discusión como formación discursiva; es ocuparse no de las contradicciones formales de sus proposiciones, sino del sistema de formación de sus objetos, de sus tipos de enunciaciones, de sus conceptos, de sus elecciones teóricas. Es reasumirla como práctica entre otras prácticas" (11). En eso, humildemente, estamos algunos. Espero que se nos perdone por ello. 
DEBATES

\section{BIBLIOGRAFÍA}

1.- Fernández Liria A : "De las psicopatologías críticas a la crítica de la psicopatología”. Rev. Asoc Esp. de Neuropsiquiatría. 2001, 21(80): 1479-1482.

2.- Fernández Liria, A : "Diagnóstico y realidad". Psiquiatría Pública. 1997, 9 (1): 51-54.

3.- Kraepelin E : Hundert Jahre Psychiatrie. Trad, Esp.: Cien años de psiquiatría. AEN. Madrid. 1999.

4.- Gaupp R : Zur Psychologie des Massenmords (Der Fall Wagner). 1914. Trad. Esp : El Caso Wagner. AEN. Madrid. 1998.

5.- Schneider K: Klinische Psychopathologie. 1946. Trad. Esp.: Psicopatología Clínica. Fundación Archivos de Neurobiología. Madrid. 1997.

6.- Ey H, Bernard P, Brisset CH : Manuel de Psychiatrie. Trad. Esp.: Tratado de Psiquiatría. Toray Masson (8 ${ }^{a}$ Edición). Barcelona. 1978.

7.- Castilla del Pino C : Introducción a la Psiquiatría. Alianza Universidad. Textos. Madrid. 1979.

8.- Díez Patricio A: "La psicogénesis del delirio en la obra y en la época de E. Kretschmer”. Rev. Asoc Esp. de Neuropsiquiatría. 2001, 21(80): 1495-1519.

9.- Alvarez JM, Colina F (eds.): El delirio en la clínica francesa. De Lasègue a Clééràmbault. Madrid. Ediciones Dor. SL. 1994.

10.- Retolaza A. "Psiquiatría Pública como método científico". Psiquiatría Pública, 1993, 5 (5) : 271-281.

11.- Foucault M: La Arqueología del saber. Madrid. Siglo XXI (5ª Edición), 1978.

\footnotetext{
* Médico psiquiatra

-Responsable-Jefe del Centro de Salud Mental de Basauri-Galdakao

-Servicios de Salud Mental y Asistencia Psiquiátrica de Bizkaia

-Osakidetza/Servicio Vasco de Salud

Dirección para correspondencia:

CSM de Basauri

C/Nagusia s.n.

48970-Basauri (Bizkaia)

Tfn.: 946007343

Fax.: 946007342

correo electrónico : basauri@smeb.osakidetza.net

Fecha de recepción: 20-03-02
} 